\title{
Non-intubated video-assisted thoracic surgery for subxiphoid anterior mediastinal tumor resection
}

\author{
Yong Mao $^{1,2 \#}$, Hengrui Liang ${ }^{2 \#}$, Shiqi Deng ${ }^{3 \#}$, Yuan Qiu ${ }^{2}$, Yanran Zhou ${ }^{4}$, Hanzhang Chen ${ }^{2}$, Long Jiang ${ }^{2}$, \\ Jianxing $\mathrm{He}^{2}$
}

${ }^{1}$ Department of Cardiothoracic Surgery, Ningbo First Hospital, Ningbo, China; ${ }^{2}$ Department of Thoracic Surgery and Oncology, the First Affiliated Hospital of Guangzhou Medical University, State Key Laboratory of Respiratory Disease, National Clinical Research Center for Respiratory Disease, Guangzhou Institute of Respiratory Health, Guangzhou, China; ${ }^{3}$ Department of Anesthesia, Guangzhou Women and Children's Medical Center, Guangzhou Medical University, Guangzhou, China; ${ }^{4}$ Department of Anesthesia, the First Affiliated Hospital of Guangzhou Medical University, Guangzhou, China

Contributions: (I) Conception and design: Y Mao, H Liang, J He; (II) Administrative support: J He, H Chen, L Jiang; (III) Provision of study materials: Y Mao, H Liang, S Deng, H Chen; (IV) Collection and assembly of data: Y Mao, Y Zhou, Y Qiu; (V) Data analysis and interpretation: All authors; (VI) Manuscript writing: All authors; (VII) Final approval of manuscript: All authors.

\#These authors contributed equally to this work.

Correspondence to: Long Jiang, MD; Jianxing He, MD. Department of Thoracic Surgery and Oncology, the First Affiliated Hospital of Guangzhou Medical University, State Key Laboratory of Respiratory Disease, National Clinical Research Center for Respiratory Disease, Guangzhou Institute of Respiratory Health, Guangzhou, China. Email: drjiang_long@163.com; drjianxing.he@gmail.com.

Background: Subxiphoid approach for mediastinal tumor resection was reported to provide a better view and less postoperative pain. Non-intubated video-assisted thoracic surgery (NI-VATS) without muscle relaxant would decrease the possibility of postoperative airway collapse for anterior mediastinal mass operation. Herein, we sought to describe the use of NI-VATS through subxiphoid approach for anterior mediastinal tumor resection.

Methods: In this retrospective cohort study, patients that underwent subxiphoid VATS resection for anterior mediastinal tumor between December 2015 and September 2019 were divided into two groups: NIVATS and intubated VATS (I-VATS). Intraoperative and postoperative variables were compared.

Results: A total of 40 patients were included. Among them, 21 patients received NI-VATS (52.5\%) and 19 were treated with I-VATS (47.5\%). In total, intraoperative (4/21 vs. 2/19; $\mathrm{P}=0.446)$ and postoperative complications (5/21 vs. 7/19; $\mathrm{P}=0.369)$ were similar between NI-VATS and I-VATS group. The anesthesia time (231.76 vs. $244.71 \mathrm{~min} ; \mathrm{P}=0.218$ ), the operation time (152.35 vs. $143.64 \mathrm{~min} ; \mathrm{P}=0.980$ ), chest tube duration (1.81 vs. 1.84 days; $\mathrm{P}=0.08)$, the total volume (351.95 vs. $348.00 \mathrm{~mL} ; \mathrm{P}=0.223)$, post-operative pain scores (2.79 vs. 2.93, $\mathrm{P}=0.413)$, and the length of stay (9.47 vs. 10.57 days; $\mathrm{P}=0.970)$ were all comparable between two groups.

Conclusions: NI-VATS for mediastinal tumor resection via subxiphoid approach is a safe and technically feasible option in selected patients, which leads to comparable perioperative clinical outcomes when compared with I-VATS via the subxiphoid approach. This technique could be used as an alteration when intubation is not available.

Keywords: Non-intubated video-assisted thoracic surgery (NI-VATS); subxiphoid; mediastinal tumor

Submitted Aug 26, 2020. Accepted for publication Dec 18, 2020.

doi: $10.21037 /$ atm-20-6125

View this article at: http://dx.doi.org/10.21037/atm-20-6125 


\section{Introduction}

Surgical resection is the main treatment for mediastinal neoplasms, both in malignant and benign tumors. Conventional median sternotomy is considered standard therapy in the last century, but video-assisted thoracic surgery (VATS) has gradually taken place in decades (1). The Transthoracic approach is the most common choice via VATS; however, the subxiphoid approach for anterior mediastinal tumor resection was reported to provide an excellent view of the bilateral pleural cavities be associated with a reduction of the postoperative pain compared with Transthoracic approach (2).

General anesthesia might increase the postoperative risks for anterior mediastinal tumor resection (3). The most feared complication is the airway collapse after intubation and muscle relaxation, potentially causing prolonged extubation after surgery $(4,5)$. Several studies have demonstrated that non-intubated VATS (NI-VATS) might solve this problem without using muscle relaxant and intubation (3).

However, NI-VATS is rarely reported in the subxiphoid approach for mediastinal tumor patients, which would limit the use of subxiphoid surgery when surgeon performing NIVATS. In this study, we reported our initial experience and explored the feasibility and safety of NI-VATS management in anterior mediastinal tumor resection via subxiphoid approach.

We present the following article in accordance with the STROBE reporting checklist (available at http://dx.doi. org/10.21037/atm-20-6125).

\section{Methods}

\section{Study design and patient inclusion}

This is a retrospective cohort study. The data of all patients who underwent subxiphoid anterior mediastinal tumor resection at the first affiliated hospital of Guangzhou Medical University between December 2015 and September 2019 were identified and consecutively collected through electronic medical records. The study was conducted in accordance with the Declaration of Helsinki (as revised in 2013). The institutional ethics committee of the hospital reviewed the study protocol and methods (2018-57). Informed consent was obtained from every patient. Exclusion criteria: subjects that underwent median sternotomy, subjects that underwent Transthoracic approach surgery, malignant cases with evident invasion to the surrounding organs. The thoracic surgeons evaluated all patients before operation. Computed tomography (CT) scans of all patients were performed to confirm the lesions' size and position by two independent radiologists. All patients who met the criteria were asked whether they would like to receive a new technique of NI-VATS; Patients who refused would get the conventional intubated surgery. Before surgery, anesthetists visited each patient to judge who could be potentially received non-intubated surgery. Patients who underwent NIVATS comprised the experimental group, and patients who received intubated VATS (I-VATS) comprised the control group.

Contraindications to NI-VATS: American Society of Anesthesiologists (ASA) class 3 or greater, body mass index (BMI) 30 or greater, severe heart disease (e.g., ischemia, arrhythmia), hemodynamic instability, coagulopathy, asthma, and sleep apnea syndrome (6).

\section{Surgical procedures}

The surgical process is the same between NI-VATS and I-VATS groups via the mediastinal approach (Figure 1). Details of the surgical procedure have already been reported elsewhere (7). A $3-\mathrm{cm}$ incision was made $2 \mathrm{~cm}$ below the lower edge of the xiphoid for setting the thoracoscope. Two 5-mm extra pleural thoracic ports were created at the midclavicular line intersecting with the bilateral costal arch to introduce thoracoscopic grasping forceps and a harmonic scalpel. A pneumomediastinum was created by an $8 \mathrm{cmH}_{2} \mathrm{O}$ positive pressure carbon dioxide $\left(\mathrm{CO}_{2}\right)$ insufflation to enlarge the retrosternal space and facilitate the tumor's dissection. Both the right-sided and left-sided mediastinal pleura were opened. The incision in the chest wall could cause the collapse of the lungs. The tumor and surrounding tissue were resected and removed through the subxiphoid port. Finally, the air in the chest cavities was evacuated by inflating the lungs. A drainage tube was inserted into the mediastinum through the subxiphoid incision. If necessary, the process was changed from the subxiphoid approach to the Transthoracic approach or open thoracotomy.

\section{Anesthesia management}

I-VATS: sufentanil $0.3-0.6 \mu \mathrm{g} / \mathrm{kg}$, target-controlled infusion (TCI) of propofol plasma concentration of $2.0-3.0 \mu \mathrm{g} / \mathrm{mL}$, and cis-atracurium $0.2 \mathrm{mg} / \mathrm{kg}$ were injected intravenously for induction. A double-lumen tube (DLT) was inserted, the placement of the bronchial tube was affirmed by 

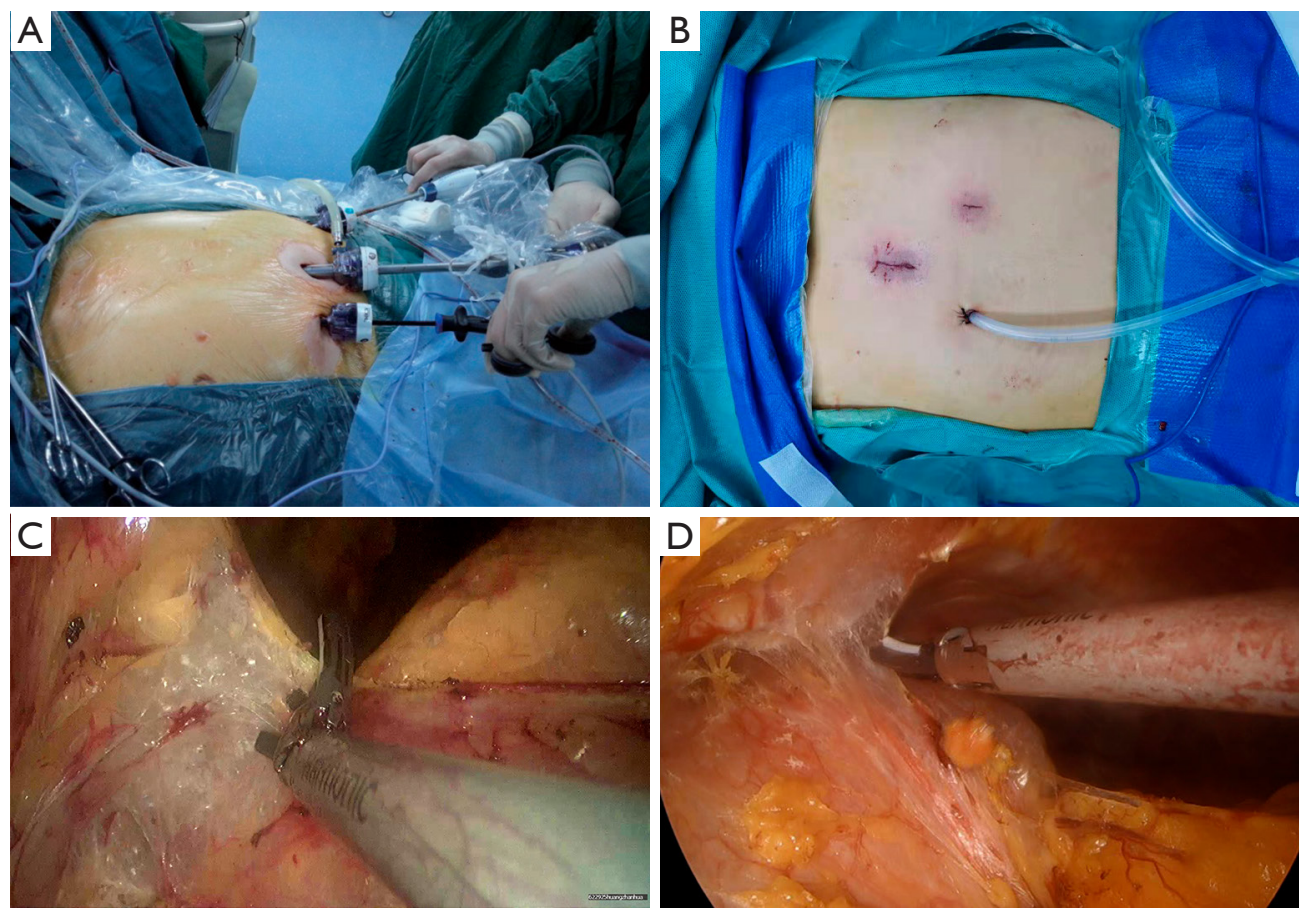

Figure 1 Surgery procedure of non-intubated video-assisted thoracoscopic surgery for mediastinal tumor resection via subxiphoid approach. (A) Approach portal location of subxiphoid video-assisted thoracoscopic surgery; (B) post-operative chest tube location; (C) opening left pleural cavity; (D) opening right pleural cavity.

bronchoscopy. For anesthesia maintain, inhaled sevoflurane 0.8-1.5 times minimum alveolar concentration (MAC), TCI of propofol plasma concentration $0.5-1.0 \mu \mathrm{g} / \mathrm{mL}$, remifentanil $0.05-0.15 \mu \mathrm{g} / \mathrm{kg} / \mathrm{min}$, dexmedetomidine $0.05-0.10 \mu \mathrm{g} / \mathrm{kg} / \mathrm{min}$ and cis-atracurium $0.2 \mathrm{mg} / \mathrm{kg} / \mathrm{h}$ were administrated. One lung ventilation was applied during the surgery; ventilation parameters were adjusted as tidal volume (VT) $6-8 \mathrm{~mL} / \mathrm{kg}$, respiration rate (RR) 10-15 times/min in volume-controlled ventilation (VCV) mode. The lung recruitment maneuvers were performed after operation completion, maintaining the maximum artificial pressure not exceeding $20 \mathrm{cmH}_{2} \mathrm{O}$ for 5 seconds. The neostigmine and atropine were routinely given for antagonism; the patient's respiratory rate, tidal volume, and $\mathrm{SpO}_{2}$ were carefully observed to reach the clinical stage.

NI-VATS: sufentanil $0.1-0.3 \mu \mathrm{g} / \mathrm{kg}$ and TCI of propofol plasma concentration $2.0-3.0 \mu \mathrm{g} / \mathrm{mL}$ were injected intravenously. Muscle relaxants were not used in the entire process of surgery. A laryngeal mask (FORNIA, Disposable Laryngeal Mask) was placed after anesthesia induction. For anesthesia maintain, TCI of more propofol plasma concentration $2-3 \mu \mathrm{g} / \mathrm{mL}$, remifentanil $0.05-0.15 \mu \mathrm{g} / \mathrm{kg} / \mathrm{min}$, and dexmedetomidine $0.05-0.10 \mu \mathrm{g} / \mathrm{kg} / \mathrm{min}$ were administrated. During the surgery, ventilation parameters were adjusted VT 3-8 mL/kg, RR 10-15 times/min in synchronized intermittent mandatory ventilation (SIMV) mode.

If hypoxemia or hypercapnia, or other indicated conditions for conversion occur during the surgery and cannot be resolved after non-invasive management, the anesthesiologist must switch the anesthesia mode and perform tracheal intubation. The single lumen endotracheal tube is preferred.

The single-lumen tube should be inserted under the guidance of a fiber optic bronchoscope when the patient is in a lateral position, which is more difficult than normal practice. To achieve this a small pillow should be placed under the head to allow the front, bottom perspective of the mouth and nose to stay up and the head and neck should be parallel to the central axis of the body.

\section{Data collection and statistical analyses}

Baseline characteristics including age, BMI, gender, tumor size, histology, and myasthenia gravis status were extracted 


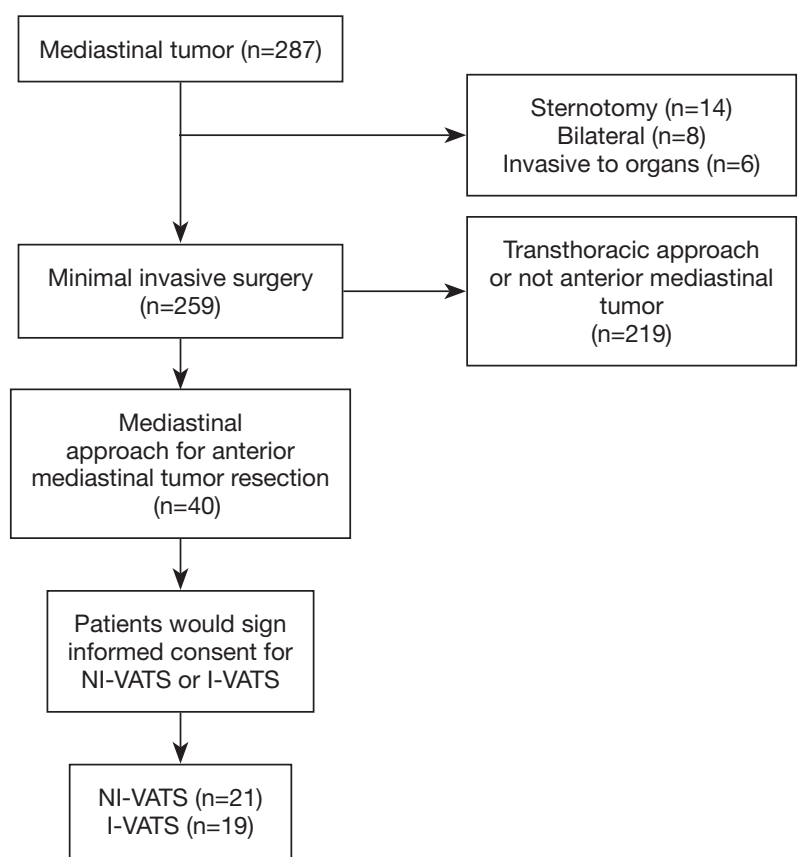

Figure 2 Schema of patient grouping and matching. I-VATS, intubated video-assisted thoracoscopic surgery; NI-VATS, non-intubated video-assisted thoracoscopic surgery.

to observe the balance between the two groups. The clinical data included intraoperative blood loss, anesthesia time, operation time, hospital duration after surgery, postoperative chest tube duration, postoperative drainage volume, and pain evaluation. Continuous data are presented as mean and standard deviation and were analyzed with 2 -sample Student $t$-tests for independent data. Categorical variables are given as a count and percentage of patients and compared with the $\chi^{2}$ or Fisher exact test. All statistical tests were 2 -sided. $\mathrm{P}$ values of less than 0.05 were considered significant. SPSS software (SPSS version 25.0; IBM Corp., Armonk, NY) was used for all data analysis (8).

\section{Results}

\section{Patients characteristics}

A total of 40 patients between December 2015 and September 2019 who underwent subxiphoid VATS anterior mediastinal tumor resection were consecutively included in this analysis (Figure 2). Among them, 21 patients received NI-VATS $(52.5 \%)$ and, 19 patients were treated under I-VATS (47.5\%). The baseline characteristics were summarized in Table 1. Baseline demographic and clinical variables were well balanced between the two groups.

\section{Operative results and postoperative recovery}

No surgery-related deaths occurred. In total, operative/ anesthesia-related intraoperative complications were similar in NI-VATS groups and I-VATS (2/21 vs. 4/19; $\mathrm{P}=0.446)$. Specifically, two patients in the I-VATS group and one patient in the NI-VATS group were converted to the Transthoracic approach due to an excess of fat tissues. Two patients in the I-VATS group and one patient in the NIVATS group were switched to median sternotomy due to the pleural adhesions.

Post-operative complication incidence was less in the NI-VATS group, although without significant statistical (5/21 vs. 7/19; $\mathrm{P}=0.369)$. No nerve damage or prolonged extubation owing to airway collapse occurred in both groups. All perioperative complications were summarized in Table 2.

The anesthesia time (231.76 vs. $244.71 \mathrm{~min} ; \mathrm{P}=0.218$ ), the operation time (152.35 vs. $143.64 \mathrm{~min} ; \mathrm{P}=0.980)$, operative bleeding (34.74 vs. $38.47 \mathrm{~mL} ; \mathrm{P}=0.267)$ and the length of stay (9.47 vs. 10.57 day; $\mathrm{P}=0.970$ ) were all similar between the two NI-VATS and I-VATS group. Chest tube duration was similar in both groups (1.81 vs. 1.84 days; $\mathrm{P}=0.08)$, and the total volume (351.95 vs. $348.00 \mathrm{~mL}$; $\mathrm{P}=0.223$ ) was also similar in the two groups. 
Table 1 Demographic characteristics of study

\begin{tabular}{|c|c|c|c|c|c|}
\hline \multirow{2}{*}{ Characteristics } & \multicolumn{2}{|c|}{ NI-VATS } & \multicolumn{2}{|c|}{ I-VATS } & \multirow{2}{*}{$P$} \\
\hline & $n=21$ & $\mathrm{SD} / \%$ & $n=19$ & $\mathrm{SD} / \%$ & \\
\hline Age (year), mean & 43.90 & 15.18 & 54.26 & 11.64 & 0.16 \\
\hline Gender & & & & & 0.751 \\
\hline Male & 11 & $52.38 \%$ & 10 & $52.63 \%$ & \\
\hline Female & 10 & $47.62 \%$ & 9 & $47.37 \%$ & \\
\hline BMI $\left(\mathrm{kg} / \mathrm{m}^{2}\right)$, mean & 23.01 & 3.64 & 23.49 & 2.52 & 0.12 \\
\hline FVC (L), mean & 3.55 & 0.79 & 3.16 & 0.94 & 0.38 \\
\hline FEV1 (L), mean & 2.86 & 0.68 & 2.46 & 0.87 & 0.24 \\
\hline ASA status class & & & & & 0.52 \\
\hline I & 10 & $47.62 \%$ & 11 & $57.89 \%$ & \\
\hline II & 11 & $52.38 \%$ & 8 & $42.11 \%$ & \\
\hline $\begin{array}{l}\text { Tumor size }(\mathrm{cm}) \text {, } \\
\text { mean }\end{array}$ & 4.81 & 1.49 & 5.26 & 1.56 & 0.24 \\
\hline Myasthenia gravis & & & & & 0.92 \\
\hline Yes & 2 & $9.52 \%$ & 2 & $10.53 \%$ & \\
\hline None & 19 & $90.48 \%$ & 17 & $89.47 \%$ & \\
\hline Pathology & & & & & 0.16 \\
\hline Thymoma & 10 & $47.62 \%$ & 5 & $26.31 \%$ & \\
\hline Cysts & 6 & $28.67 \%$ & 4 & $21.05 \%$ & \\
\hline Fibroma & 5 & $23.71 \%$ & 10 & $52.64 \%$ & \\
\hline
\end{tabular}

NI-VATS, non-intubated video-assisted thoracic surgery; I-VATS, intubated video-assisted thoracic surgery; BMI, body mass index; FVC, forced vital capacity; $F E V_{1}$, forced expiratory volume in 1 second; ASA, American Society of Anesthesiologists; SD, standard deviation.

\section{Postoperative analgesia medication}

Comprehensive data of 31 patients (17 NI-VATS, 14 I-VATS) were available for the post-operative pain score analysis. The visual analogue scale score was evaluated on the postoperative day one of each patient. A similar level of VAS score was observed in the NI-VATS group compared with the I-VATS group $(2.79 \pm 1.42$ vs. $2.93 \pm 1.83, \mathrm{P}=0.413)$. Besides, the patient number of using postoperative opioid analgesia was similar in the NI-VATS group compared with the I-VATS groups $(17.64 \%$ vs. $29.41 \%, \mathrm{P}=0.368)$

\section{Discussion}

This is the first cohort study on subxiphoid NI-VATS
Table 2 Perioperative complications between NI-VATS group and I-VATS group

\begin{tabular}{|c|c|c|c|c|}
\hline \multirow{2}{*}{ Variables } & \multicolumn{2}{|c|}{ NI-VATS $(n=21)$} & \multicolumn{2}{|c|}{ I-VATS $(n=19)$} \\
\hline & $\mathrm{n}$ & $\%$ & $\mathrm{n}$ & $\%$ \\
\hline \multicolumn{5}{|l|}{ Intraoperative complications } \\
\hline Total & 2 & 9.5 & 4 & 21.1 \\
\hline $\begin{array}{l}\text { Converted to the trans } \\
\text { thoracic approach }\end{array}$ & 1 & 4.8 & 2 & 10.5 \\
\hline $\begin{array}{l}\text { Converted to median } \\
\text { sternotomy }\end{array}$ & 1 & 4.8 & 2 & 10.5 \\
\hline \multicolumn{5}{|l|}{ Postoperative complications } \\
\hline Total & 5 & 23.8 & 7 & 36.8 \\
\hline Fever & 2 & 9.5 & 1 & 5.3 \\
\hline Vomiting & 0 & 0 & 1 & 5.3 \\
\hline Dizziness & 1 & 4.8 & 1 & 5.3 \\
\hline Sore throat & 0 & 0 & 3 & 15.8 \\
\hline Incision pain & 4 & 19.0 & 4 & 21.1 \\
\hline Dyspnea & 0 & 0 & 1 & 5.3 \\
\hline Total perioperative complications & 7 & 33.3 & 11 & 57.9 \\
\hline
\end{tabular}

NI-VATS, non-intubated video-assisted thoracic surgery; I-VATS, intubated video-assisted thoracic surgery.

mediastinal tumor resection, which indicated that NIVATS was a safe and feasible procedure via the subxiphoid approach. Although statistically significant was not observed because of the small sample size, NI-VATS might potentially decrease perioperative complications compared with I-VATS (7/21 vs. 11/19). It can be considered an alternative to I-VATS when the lateral approach is not available or when surgeons prefer subxiphoid surgery.

Compared with the Transthoracic approach, the most prominent advantages of the subxiphoid approach for mediastinal tumors are more visual and less postoperative pain (9). Complete resection of the tumor is the primary aim. With the help of a camera scope inserted from the midline of the body, it would be convenient to look over the bilateral mediastinum clearly and confirm the location of important blood vessels and nerves nearby (10). However, there are still some shortcomings of the subxiphoid approach. First, a surgical team familiar with the subxiphoid approach procedure is needed. Second, it's hard to play when severe adhesions are existing. Third, the tumor size also needs to be limited because of the narrow space (7). 
Therefore, the application of the subxiphoid approach may be restricted in some conditions.

One of our recent work demonstrated that the subxiphoid approach is associated with less pain compared with the intercostal approach. The right intercostal thoracic approach may offer a better clinical effect of short-term postoperative recovery (11). When the tumor was located at the thoracic inlet, the transcervical approach was selected. Migliore et al. (12). has described their experience in single incision extended video-assisted transcervical thymectomy and demonstrated that this is a safe and successful procedure. However, we have not tried such procedure under non-intubated anesthesia.

The I-VATS group's anesthesia process in subxiphoid approach is almost the same as it in other thoracic surgeries (13). When using a DLT for one-lung ventilation, contralateral lung collapse is easy to achieve via gentle press by a surgeon and air suction by an anesthesiologist. Nonetheless, the anesthesia process of the NI-VATS group under the subxiphoid approach is a novel attempt. There was only one case report about subxiphoid NI-VATS for thymectomy in 2017; the patient was selected carefully, indicating that NI-VATS in the subxiphoid approach was still under exploration (14). The present study revealed that NI-VATS under subxiphoid approach was a feasible procedure with similar intraoperative and postoperative outcomes with conventional I-VATS group.

Spontaneous ventilation during surgery relies on the integrality of at least one side of the pleura. The three-port subxiphoid approach in our study cuts bilateral mediastinal pleura, causing the collapse of both sides of lungs, which made complete spontaneous ventilation impossible. When there is bilateral pneumothorax simultaneously, the mediastinal swing would stop, followed by a continuous deflation and inflation of bilateral lungs, which would bring a great challenge to the surgeon. To alleviate this problem, we adopted a small tide volume (3-4 L/min) ventilation through SIMV mode, which would maintain the oxygen supply and minimize the impact on the operation.

Non-intubated anesthesia management for the subxiphoid approach includes laryngeal mask airway general anesthesia (without muscle relaxant) in SIMV mode, combined with vagus and intercostal nerve blocking. The laryngeal mask could potentially avoid intubationassociated complications, including postoperative sore throat, hoarseness, and irritating cough. Besides, the problematic intubation is not uncommon in thymoma patients, rendering a laryngeal mask the preferred option
$(6,15)$. We have done the thymus horns and the perihilar fat in thymoma patients between the phrenic nerves at both sides in NI-VATS and I-VATS groups. When dissecting fat, surgeons need to be careful not to injure the phrenic nerves. After the vagus nerve blocked by lidocaine, the cough almost disappeared. For mediastinum moving, a little dose of cis-atracurium would significantly reduce it.

Not using muscle relaxants is a benefit for myasthenia gravis patients, which can avoid the residual effects of muscle relaxants, achieve faster recovery of respiratory muscle function, and lower the incidence of difficult postoperative extubation (16). The benefits of omitting muscle relaxants are not limited in myasthenia gravis patients; it was reported that the use of muscle relaxants was associated with an increased risk of postoperative pulmonary complications, which cannot be reduced by the administration of reversal agents like sugammadex (17). In addition, during large anterior mediastinal tumor resection, muscle relaxants would cause airway collapse, NI-VATS would potentially avoid this situation (18). Although no airway collapse or prolonged extubation case occurred in our study, it could happen when the mediastinal mass is big. One of the important meanings of this study is to prove the feasibility and safety of NI-VATS under the subxiphoid approach. When surgeons confront a situation when convention I-VATS may have a high risk of causing airway collapse, he/she could also use NI-VATS via a subxiphoid approach.

There are several limitations in our study, including a small sample size, short follow-up, and retrospective nature. Because the process is relatively novel, the steps are unfamiliar, and good cooperation is needed between the surgeons and the anesthesiologists, this new approach was not commonly performed. The morbidity of the anterior mediastinal tumor was low and subxiphoid surgery was not routine in thoracic surgery; this study reported a preliminary exploration of non-intubated subxiphoid VATS for mediastinal tumor, with the aim to confirm its feasibility. According to this target, this study has achieved this goal. A lack of intra-operative ventilation information (hypoxaemia and hypercapnia) hinders our further understanding of perioperative pneumodynamics.

\section{Conclusions}

In summary, this study reveals that NI-VATS mediastinal tumor resection is a safe and technically feasible option in selected patients. This technique leads to comparable 
postoperative outcomes when compared with I-VATS.

\section{Acknowledgments}

Funding: This work was supported by Bethune Ethicon Excellence Surgery Fund (2018).

\section{Footnote}

Reporting Checklist: The authors have completed the STROBE reporting checklist. Available at http://dx.doi. org/10.21037/atm-20-6125

Data Sharing Statement: Available at http://dx.doi. org/10.21037/atm-20-6125

Peer Review File: Available at http://dx.doi.org/10.21037/ atm-20-6125

Conflicts of Interest: All authors have completed the ICMJE uniform disclosure form (available at http://dx.doi. org/10.21037/atm-20-6125). JH serves as an unpaid Editors-in-Chief of Annals of Translational Medicine. The other authors have no conflicts of interest to declare.

Ethical Statement: The authors are accountable for all aspects of the work in ensuring that questions related to the accuracy or integrity of any part of the work are appropriately investigated and resolved. The study was conducted in accordance with the Declaration of Helsinki (as revised in 2013). The study protocol and methods were reviewed by the institutional ethics committee of the First Affiliated Hospital of Guangzhou Medical University (2018-57). Informed consent was obtained from every patient.

Open Access Statement: This is an Open Access article distributed in accordance with the Creative Commons Attribution-NonCommercial-NoDerivs 4.0 International License (CC BY-NC-ND 4.0), which permits the noncommercial replication and distribution of the article with the strict proviso that no changes or edits are made and the original work is properly cited (including links to both the formal publication through the relevant DOI and the license). See: https://creativecommons.org/licenses/by-nc-nd/4.0/.

\section{References}

1. Rascoe PA, Kucharczuk JC, Cooper JD. Surgery of the mediastinum: historical notes. Thorac Surg Clin 2009; 19:1-5.

2. Yano M, Moriyama S, Haneda H, et al. The Subxiphoid Approach Leads to Less Invasive Thoracoscopic Thymectomy Than the Lateral Approach. World J Surg 2017;41:763-70.

3. Jiang L, Depypere L, Rocco G, et al. Spontaneous ventilation thoracoscopic thymectomy without muscle relaxant for myasthenia gravis: Comparison with "standard" thoracoscopic thymectomy. J Thorac Cardiovasc Surg 2018;155:1882-9.e3.

4. Slinger P, Karsli C. Management of the patient with a large anterior mediastinal mass: recurring myths. Curr Opin Anaesthesiol 2007;20:1-3.

5. Sendasgupta C, Sengupta G, Ghosh K, et al. Femorofemoral cardiopulmonary bypass for the resection of an anterior mediastinal mass. Indian J Anaesth 2010;54:565-8.

6. Sunaga H, Blasberg JD, Heerdt PM. Anesthesia for nonintubated video-assisted thoracic surgery. Curr Opin Anaesthesiol 2017;30:1-6.

7. Lu Q, Zhao J, Wang J, et al. Subxiphoid and subcostal arch "Three ports" thoracoscopic extended thymectomy for myasthenia gravis. J Thorac Dis 2018;10:1711-20.

8. Wu CF, Hsieh MJ, Liu HP, et al. Management of postoperative pain by placement of an intraoperative intercostal catheter after single port video-assisted thoracoscopic surgery: a propensity-score matched study. J Thorac Dis 2016;8:1087-93.

9. Suda T. Subxiphoid thymectomy: single-port, dual-port, and robot-assisted. J Vis Surg 2017;3:75.

10. Numanami H, Yano M, Yamaji M, et al. Thoracoscopic Thymectomy Using a Subxiphoid Approach for Anterior Mediastinal Tumors. Ann Thorac Cardiovasc Surg 2018;24:65-72.

11. Ardakani AA, Kanafi AR, Acharya UR, et al. Application of deep learning technique to manage COVID-19 in routine clinical practice using CT images: Results of 10 convolutional neural networks. Comput Biol Med 2020;121:103795.

12. Migliore $M$, Criscione A, Nardini $M$, et al. Single incision extended video assisted transcervical thymectomy. J Vis Surg 2017;3:154.

13. Guo Z, Yin W, Pan H, et al. Video-assisted thoracoscopic surgery segmentectomy by non-intubated or intubated anesthesia: a comparative analysis of short-term outcome. J Thorac Dis 2016;8:359-68.

14. Jiang L, Liu J, Shao W, et al. Non-intubated subxiphoid uniportal video-assisted thoracoscopic thymectomy using 
glasses-free 3D vision. J Thorac Dis 2016;8:E1602-4.

15. Organization. WH. Coronavirus disease 2019

(COVID-19): situation report- March 22, 2020. Accessed March 22, 2020.

16. Cui F, Liu J, Li S, et al. Tubeless video-assisted thoracoscopic surgery (VATS) under non-intubated, intravenous anesthesia with spontaneous ventilation and no placement of chest tube postoperatively. J Thorac Dis
2016;8:2226-32.

17. Kirmeier E, Eriksson LI, Lewald H, et al. Post-anaesthesia pulmonary complications after use of muscle relaxants (POPULAR): a multicentre, prospective observational study. Lancet Respir Med 2019;7:129-40.

18. Hartigan PM, Ng JM, Gill RR. Anesthesia in a Patient with a Large Mediastinal Mass. N Engl J Med 2018;379:587-8.

Cite this article as: Mao Y, Liang H, Deng S, Qiu Y, Zhou Y, Chen H, Jiang L, He J. Non-intubated video-assisted thoracic surgery for subxiphoid anterior mediastinal tumor resection. Ann Transl Med 2021;9(5):403. doi: 10.21037/atm-20-6125 